\title{
Industrial defect discrimination applying infrared imaging spectroscopy and Artificial Neural Networks
}

\author{
P. Beatriz Garcia-Allende, Olga M. Conde, Francisco J. Madruga, Ana M. Cubillas, Jose M. Lopez- \\ Higuera \\ Photonics Engineering Group, Universidad de Cantabria, Avda. Los Castros s/n, 39005 Santander, \\ Spain
}

\begin{abstract}
A non-intrusive infrared sensor for the detection of spurious elements in an industrial raw material chain has been developed. The system is an extension to the whole near infrared range of the spectrum of a previously designed system based on the Vis-NIR range $(400-1000 \mathrm{~nm})$. It incorporates a hyperspectral imaging spectrograph able to register simultaneously the NIR reflected spectrum of the material under study along all the points of an image line. The working material has been different tobacco leaf blends mixed with typical spurious elements of this field such as plastics, cardboards, etc. Spurious elements are discriminated automatically by an artificial neural network able to perform the classification with a high degree of accuracy. Due to the high amount of information involved in the process, Principal Component Analysis is first applied to perform data redundancy removal. By means of the extension to the whole NIR range of the spectrum, from 1000 to $2400 \mathrm{~nm}$, the characterization of the material under test is highly improved. The developed technique could be applied to the classification and discrimination of other materials, and, as a consequence of its non-contact operation it is particularly suitable for food quality control.
\end{abstract}

Keywords: Imaging Spectroscopy, Hyperspectral Spectrograph, Artificial Neural Networks (ANN), Principal Component Analysis (PCA), Automatic Classification.

\section{INTRODUCTION}

Raw material classification on different quality levels is a key role on the input stages of processing chains in many industrial sectors. This quality-control process consists in the automatic rejection of the unwanted spurious elements or "defects" that can be found intermingled with the desired raw material. Non-intrusive and non-contact procedures are required for this task. Therefore, optical techniques are very appropriate for this kind of applications. In addition, the presence and position of defects have to be determined while the mixed materials are transported by the conveyor belt in the production plant. And so, real-time operation, together with accuracy and reliability, are also demanded.

Imaging spectroscopy is an optical technique which allows the simultaneous determination of the optical spectrum components and the spatial location of an object in a surface. When the material to be characterized or identified is being transported on a conveyor belt, the spectral information can be taken across a transversal line and the movement of the conveyor belt automatically allows the scanning of the other spatial dimension. The main advantage of this technique is that the spectra of all the points in the line of vision are measured at the same time. Hence, non-invasive and real-time operation requirements are simultaneously satisfied. To acquire the information of both domains, spectral and spatial, at the same time a passive Prism-Grating-Prism (PGP) device can be used [1]. Imaging spectroscopy based on this kind of hyperspectral device has been widely used in airborne applications, and some references can be found in other fields such as industrial [2], biological [3], food [4] and fruit [5]. A non-intrusive and real-time imaging spectroscopy system capable of discriminating between wanted raw materials from the mixed unwanted materials was previously developed [6]. It was based on the Vis-NIR range of the spectrum $(400-1000 \mathrm{~nm})$ and the raw material from the tobacco industry was employed as the working material. In this field, a great variety of spurious elements such as plastics, cords cardboards, papers, textile threads, etc. can be found blended with wanted raw material, tobacco leaves. Satisfactory classification results were obtained since tested defects, except for transparent cellophanes, were distinguished from tobacco leaves. McClure's studies on tobacco leaves' spectrum demonstrate that they have important absorption peaks in 1450 and $1930 \mathrm{~nm}$ [7]. As a consequence, the performance of the defect discrimination system could be improved by

Thermosense XXX, edited by Vladimir P. Vavilov, Douglas D. Burleigh,

Proc. of SPIE Vol. 6939, 69390H, (2008)

0277-786X/08/\$18 - doi: $10.1117 / 12.770279$

Proc. of SPIE Vol. $693969390 \mathrm{H}-1$ 
extension over the whole NIR region of the spectrum and plastics could also be recognised. This requires the replacement of the previously employed PGP device. Therefore, a new system has to be designed and developed.

As well as in the Vis-NIR system, Artificial Neural Networks (ANNs) [8-9] are employed to accomplish the identification/classification/discrimination task. Despite of the change of spectral range, the amount of information provided by the imaging spectroscopic technique remains large. Therefore, intelligent criterions capable of extracting the relevant information, the so-called spectral fingerprint of the material, are also necessary. There are multiple different procedures to perform this data compression [10]. Principal Component Analysis (PCA) [11-12], which was successfully employed in the previous system, is here used again.

The paper is divided in five sections as follows: in Section 2, the working principles and the global block diagram of the system are described. Section 3 discusses the results and, finally, in Section 4 several conclusions are extracted.

\section{OPTOELECTRONIC SYSTEM}

A block diagram of the complete system is illustrated in Figure 1. Hyperspectral images, consisting of the diffuse reflectance of the material under study in the NIR range of the spectrum, are recorded by a camera through a PGP device. It is the so-called 'Spectral Image Acquisition' unit. In the 'Data Compression' block, relevant information is extracted from the images using PCA analysis. Finally, a specifically trained ANN identifies the raw material and provides decisions to enable the discrimination of the wanted raw materials against the spurious elements. A deeper description of the three blocks follows in the next lines.

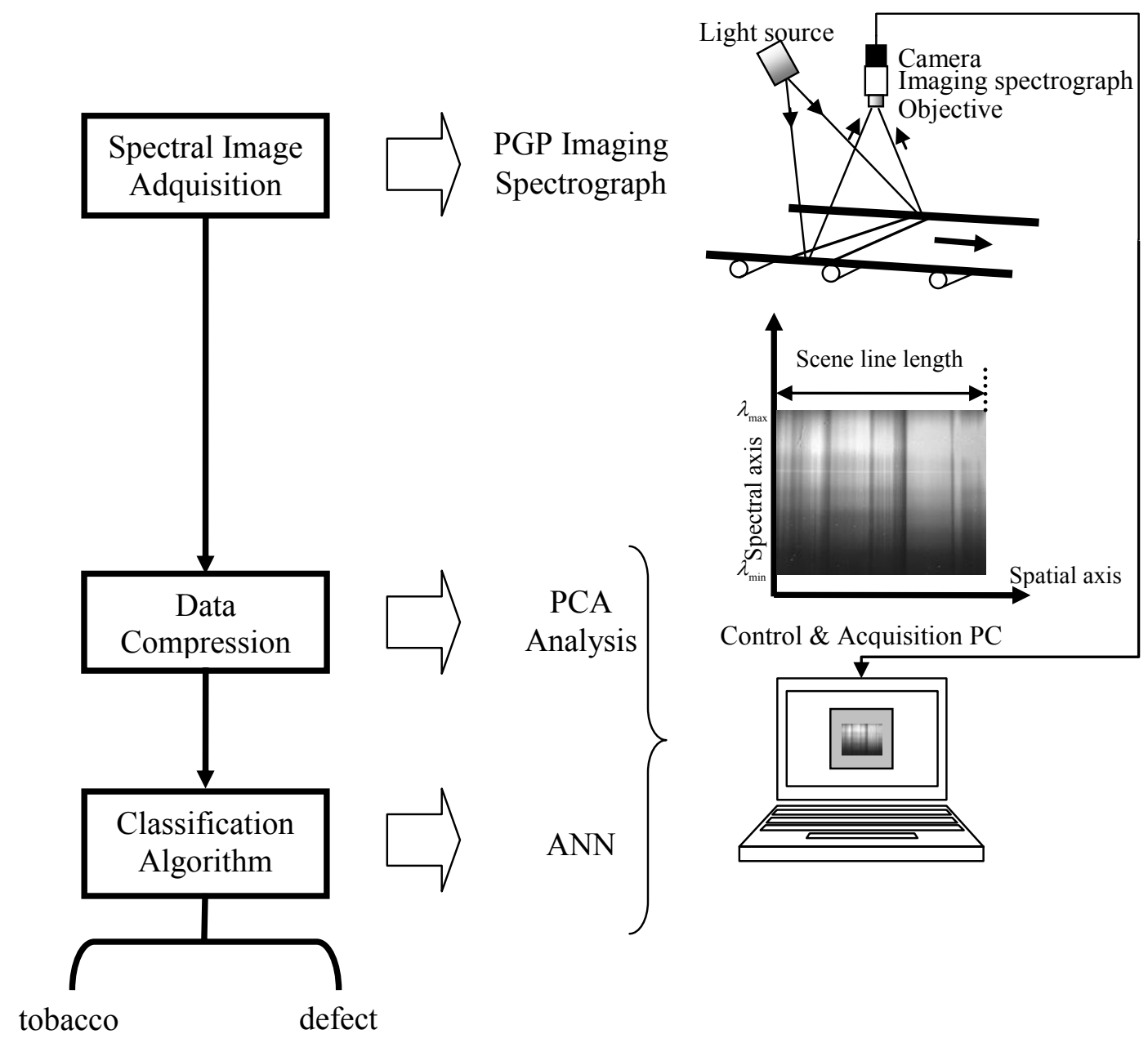

Fig. 1. Comprehensive block diagram of the classification system 


\subsection{Hyperspectral image acquisition}

The Spectral Image Acquisition unit is based on a PGP (Prism - Grating - Prism) imaging spectrograph [1]. It consists of a totally passive element connected between an objective lens and a monochrome camera with a "Camera Link" interface. Some kind of illumination system is required too. In the NIR spectroscopic system two halogen floodlights Taxley MX500, with a power rating of $500 \mathrm{~W}$ each are employed.

The main component of the acquisition system is the imaging spectrograph, since it allows the concurrent measurement of optical spectrum components and spatial location of objects. The equipment known as Imspector, commercialized by Specim Ltd., has been used. However, the V10E version used before has been replaced by the version N24E, whose spectral range goes from 1000 to $2400 \mathrm{~nm}$. As a consequence, a camera replacement is also needed. The monochrome camera XEVA-FPA-2.5-320 by Xenics, whose spectral range goes from 850 to $2500 \mathrm{~nm}$ has been utilized. Its communication interface with the control and acquisition computer is Camera Link for image transference and USB for camera control. Camera replacement implies not only a variation in the communication interface but also in system resolution. The size of the hyperspectral images is smaller in the NIR system. Camera resolution is 320 (spatial axis) $\mathrm{x}$ 256 (spectral axis). Finally, a PENTAX C1614-M (C31634) is the selected objective lens.

Before taking spectral images, several calibration processes (spatial, spectral and responsivity calibration) are demanded. The spatial calibration consists in the determination of the dimensions, length and width, of the observation line. They depend on the length and width of the slit of the Imspector, the distance between target and objective lens and the lens focal length. As the latter has been adjusted to have the target sample in focus, it was necessary to experimentally determine the dimensions. A fringe pattern was used to determine the line analyzed by the system. The wavelength calibration defines the spectral axis and it is performed using three lasers with different wavelengths within the nearinfrared range of the spectrum. Offset compensation is the measurement and subsequent subtraction of the dark current. Finally, responsivity calibration compensates detector responsitivity, lens and spectrograph throughput dependency on wavelength, light source color temperature drift and lighting spatial non-uniformity. These variations can be calibrated by measuring white and black references. For white reference Spectralon ${ }^{\circledR}$ reflectance standard is employed and the black reference is obtained by blocking the sensor input. After that, each time the system gets an image from a sample, its reflectance should be corrected as indicated in (1).

$$
R\left(\lambda_{i}\right)=\frac{S\left(\lambda_{i}\right)-D\left(\lambda_{i}\right)}{W\left(\lambda_{i}\right)-D\left(\lambda_{i}\right)}
$$

where $S\left(\lambda_{i}\right)$ is the sample value at a wavelength $\lambda_{i} ; D\left(\lambda_{i}\right)$ is the value for the dark reference and $W\left(\lambda_{i}\right)$ the equivalent for the white reference.

Table 1summarizes the main features of the measurement system.

Table 1. Summary of the main system specifications

\begin{tabular}{l|l}
\hline Spectral range (Imspector N24E) & $1000-2400 \mathrm{~nm}$ \\
Spectral resolution & $8 \mathrm{~nm}$ \\
Length of the scene line & $143 \mathrm{~mm}$ \\
Spatial resolution & $446.875 \mu \mathrm{m}$
\end{tabular}

\subsection{Data compression block}

The spectral axis of the hyperspectral images contains redundant information for the training of the ANN. Redundancy removal and data compression are performed simultaneously by means of PCA, and it consists in expressing the spectrum of each spatial pixel of the image in a different vectorial basis. The new basis vectors are those directions of the initial data which contain the most relevant information. Because of the assumption of PCA that variance means information, the first step of the analysis is to subtract the mean data. After mean subtraction, the covariance matrix of the data is computed and their eigenvalues and eigenvectors are obtained. A more detailed description of PCA application to hyperspectral images can be found in our previous work [6]. Data compression is performed neglecting those eigenvalues smaller than a thousandth of the maximum eigenvalue. In this way, the spectral axis, which was initially a 256-dimensional space, is reduced to 9 wavelengths. Therefore, a compression rate of $96.48 \%$ is obtained. A 
great enhancement in the computational performance is allowed by this reduction and the raw material classification can be carried out in real-time without causing an appreciable increase in the error probability.

In addition to spectral axis compression, an average in the spatial axis is also done in this unit. This can be done because the system of mechanical extraction of unwanted material has low spatial selectivity. Data compression and noise reduction are simultaneously provided by this average. The number of the spatial averaged points is 5 . This reduces the spatial positions considered from the initial 320 to the final 64 . Thus, with both spectral and spatial axis compressions, a total reduction of $99.3 \%$ is accomplished.

\subsection{Classification unit}

The ability of ANNs to handle non-linearity, their parallel processing of information and their quick adaptability to system dynamics were very useful when performing defect discrimination in the hyperspectral images from the Vis-NIR range of the spectrum. Therefore, a neural network able to accomplish raw material classification from its diffuse reflectance in the NIR range has been developed.

A multilayer feed-forward network and back-propagation learning algorithm have been selected. The error considered in the training phase is defined as the mean square error between the current outputs of the ANN and the desired outputs for the training spectra (' 1 ' for tobacco reflectance spectra and ' 0 ' for defect spectra). The goal error is $0.001 \%$. Different numbers of hidden layers and neurons, or processing elements, of any of them have been experimented in the test phase. The classification error percentage introduced by the ANN in the test data set is used as a measurement of the goodness of the designed net structure. Finally, the net consists in an input layer with 10 neurons and an output layer with only 1 neuron. This neuron is deactivated (' 0 ') when a defect spectrum is encountered. The neurons in all layers have a logsigmoid transfer function. Its topology is illustrated in Figure 2.

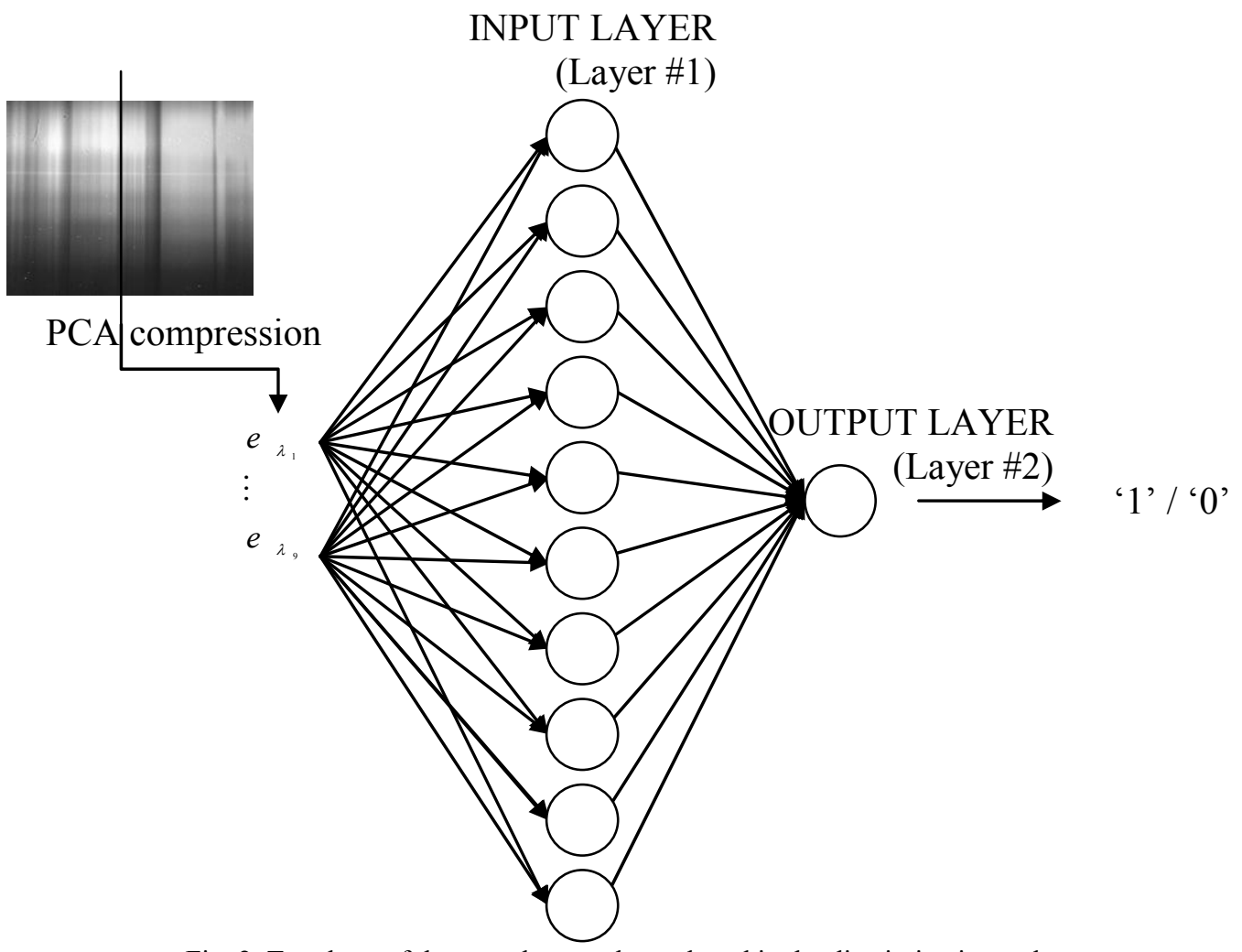

Fig. 2. Topology of the neural network employed in the discrimination task.

Training set of data consists of homogeneous samples, that is, all the pixels across the observation line correspond to the same type of material: tobacco leaves or some kind of defect, as plastic for example. The expected outputs of the network are, therefore, one (tobacco) or zero (defect) in all the spatial positions of the image. For the test phase a 
different approach has been followed: tobacco leaves and defects were combined in the same line to confirm that the system was able to spatially discriminate them. Test results will be presented and discussed in the next section.

\section{RESULTS AND DISCUSSION}

The goodness of the infrared system to perform quality control tasks was tested using tobacco samples. These were provided by the Altadis Company. These tobacco leaves are currently employed to make Havana cigars and they are in different curing states. Following the company's suggestions, the following defects or unwanted materials have been considered in order to be discriminated from tobacco leave blends: wood, cardboard, different coloured cellophanes, leather, brown and green leaves of other vegetable material different from tobacco, foil, candy paper and textile threads. These defects can be found intermingled in the tobacco leave blends due to the manual harvest procedure.

Table 2 summarizes and illustrates a subset of the analyzed samples and their corresponding results. Its columns contain the following information:

i) A photograph of the sample under analysis, where the red lines delimit the line of vision. The horizontal axis corresponds to the spatial axis of the spectrograph. After the averaging process, 64 spatial positions remain to be classified as tobacco or defect.

ii) A zoom of the scene line and a brief description of each case.

iii) The corresponding hyperspectral images. As before, the spatial axis is represented horizontally with the origin on the left as it appears in the real photograph. The spectral axis is represented vertically with the smallest wavelength of the spectral range at the bottom of the image.

iv) The last column presents the results of the classification provided by the neural network. A ' 1 ' means that that position contains tobacco meanwhile a ' 0 ' indicates that it corresponds to the presence of an unwanted material.

A special attention should be paid to sample number 8 since it showed up the improvement in the system performance obtained by the extension to the whole near infrared spectral range. The system developed in the Vis-NIR range of the spectrum was unable to correctly distinguish the transparent plastic when it was on tobacco leaves [6]. However, as shown in Table 2, this fault does not occur in the new classification system. A different type of transparent plastic is tested in sample 9 to demonstrate that the classification system is also able to detect it in a satisfying manner.

Table 2. Classification results.

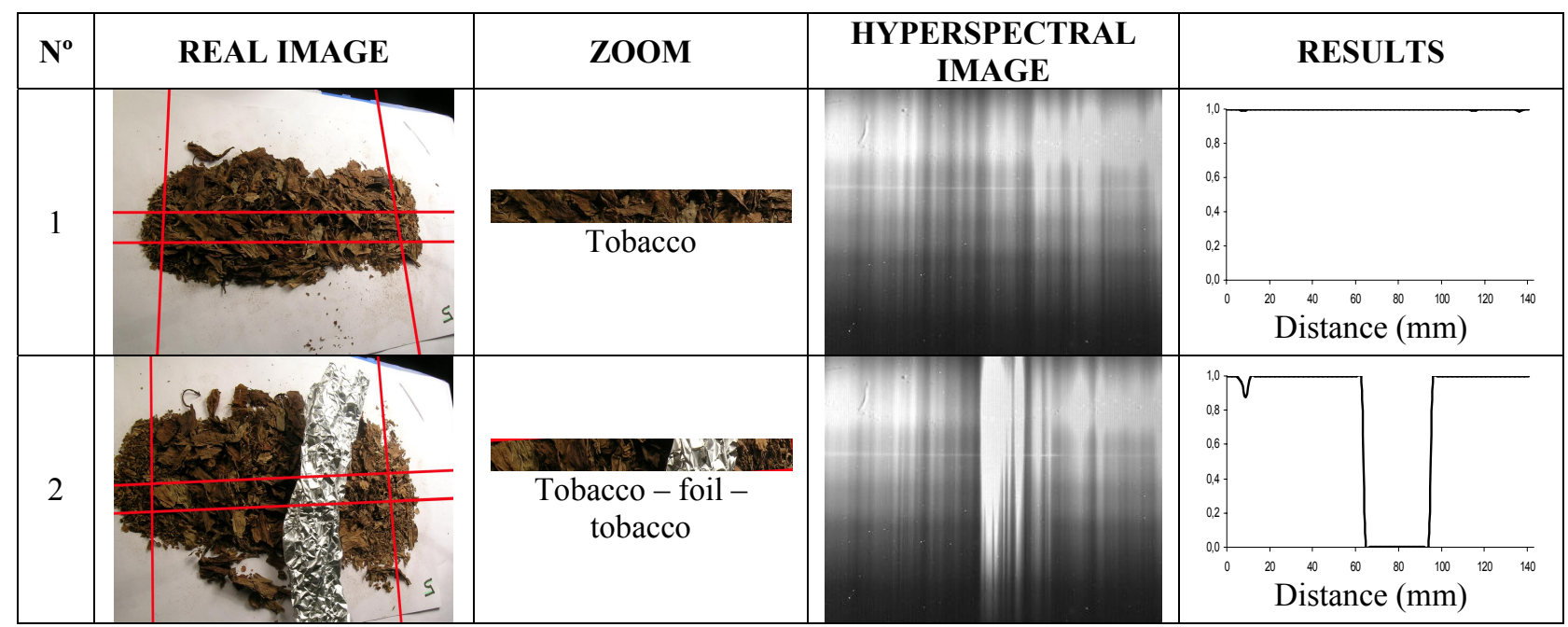




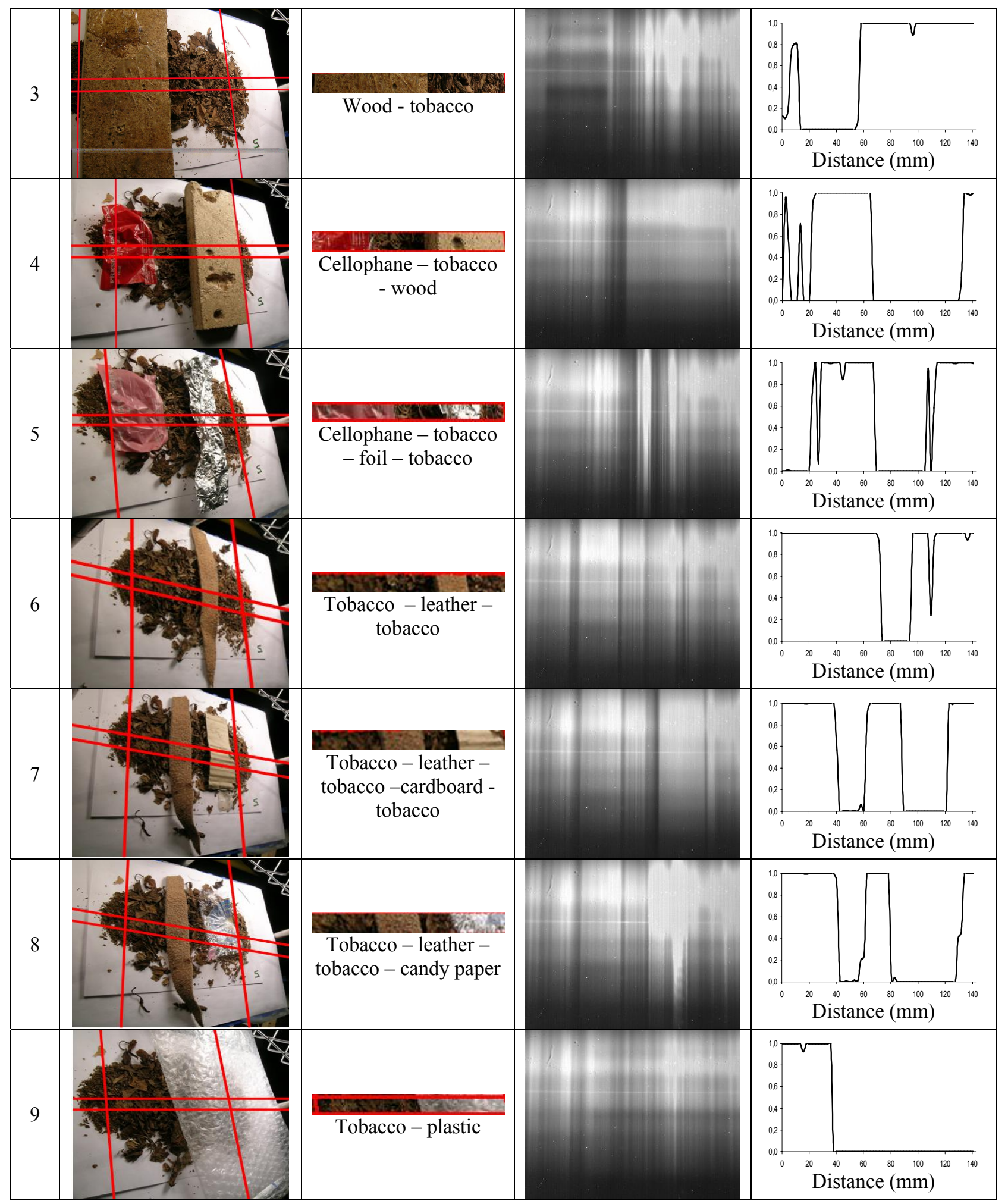




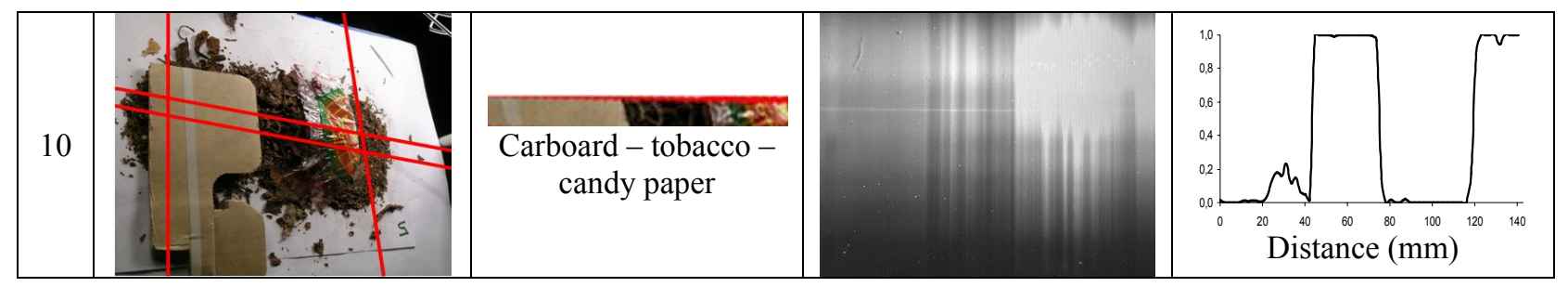

The classification results presented above are calculated by the spectral interpretation processing described in Section 2, employing 45 hyperspectral images of tobacco (2880 spectra) and 44 hyperspectral images of defects (2816 spectra) to train the neural network Complete test set consists of 60 heterogeneous hyperspectral images where tobacco leaves are therefore intermingled with all kind of defects along the line of vision. However, classification results are only presented for 10 of them because otherwise it would take too much room. This comprehensive work programme demonstrates the feasibility of the extended system to perform material classification. Table 2 also shows that several spectra are misclassified: one tobacco spectra classified as defects can be observed in samples 5 and 6, respectively. They are the socalled false alarms and some false positives, i.e. a defect spectra classified as tobacco can be seen for example in tests number 3 and 4 . The latest are the most important ones for manufacturing issues and they can be reduced to a minimum choosing a higher decision threshold, i.e. 0.8. In spite of these punctual errors, the system is able to detect the presence of the vast majority of tested defects. It is important to emphasize that, as shown in tests number 8 and 9 , the development of the system in the near infrared range of the spectrum allows the detection of transparent cellophanes and plastics that were confused with tobacco when working in the visible range [6].

\section{CONCLUSIONS}

A extension of the working spectral range of a previously developed system capable of discriminating between wanted raw materials from the mixed unwanted materials has been performed. The system is based on the imaging spectroscopy technique in the near infrared range of the spectrum, therefore the optical spectrum components from 1000 to $2400 \mathrm{~nm}$ are simultaneously measured along all the points in the vision line without the need for any scanning mechanics. This is particularly suitable when performing raw material quality control since both the presence and position of spurious elements have to be determined while the mixed materials are transported by the conveyor belt in the production plant. The performance of the system is clearly improved by this spectral extension, and defects that were previously confused with tobacco, such as transparent cellophane and plastics, are in the new range successfully detected.

As before, a specifically designed neural network has been successfully used as the spectral interpretation algorithm and the application to the hyperspectral images of the PCA analysis has been maintained to enhance the computational time performance of the ANN. Thanks to the improvement in the characterization of the material under test achieved by the extension of the spectral range, the validity of simpler classification strategies, such as the linear ones, could be investigated to reduce classification times. On the other hand, other sorting problems related with different materials could also take advantage of the extension of the spectral range of analysis, and as a consequence of its non-contact operation is particularly interesting in food quality control.

\section{ACKNOWLEDGEMENTS}

This work has been co-supported by the Science and Technology Ministry of the Spanish Government through the TEC'2005-08218-C02-02 and TEC'2007-67987-C02-01 projects. The authors also thank Infaimon Company and its staff, especially C. López and C. Carreté, for their valuable help during the arrangement of the measurement set-up. 


\section{REFERENCES}

1 T.Vaarala, M. Aikio, H.Keraenen, "An advanced prism-grating-prism imaging spectrograph in on-line industrial applications", Proc. SPIE 3101 (1997) 322-330.

${ }_{2}$ E. Herrala, T. Hyvarinen, O. Voutilainen, J. Lammasniemi, "An optoelectronic sensor system for industrial multipoint and imaging spectrometry", Sens. Actuator A-Phys. 61 (1997) 335-338.

3 G. Zavattini, S. Vecchi, R.M. Leahy, D.J. Smith, S.R. Cherry, "A hyperspectral fluorescence imaging system for biological applications", Proc. of the IEEE Nuclear Science Symposium, vol. 2, Piscataway, USA, October 19-25, 2003, pp. 942-946.

B. Park, K.C. Lawrence, W.R. Windham, D.P. Smith, P.W. Feldner, "Hyperspectral imaging for food processing automation", Proc. SPIE 4816 (2002) 308-316.

5 J. Xing, C. Bravo, T. P'al, H. Jancs'ok, J. Ramon, Baerdemaeker, "Detecting bruises on 'golden delicious' apples using hyperspectral imaging with multiple wavebands", Biosyst. Eng. 90 (2005) 27-36.

6 P.B. García-Allende, O.M. Conde, A.M. Cubillas, C. Jáuregui, J.M. López-Higuera, "New raw material discrimination system base don a spatial optical spectroscopy technique", Sens. Actuator A-Phys. 135 (2007) 605-612.

7 W.F. McClure, "Spectral characteristics of tobacco in the near-infrared region from 0.6 to 2.6 microns", Tobacco Sci. 12 (1968) 232-235.

8 C. H. Chen., Fuzzy Logic and Neural Network Handbook, McGraw Hill, New York, 1996.

9 F. Girosi, J. Makhoul, E. Manolakos and E. Wilson, "Neural Networks for Signal Processing V", Proc. of the 1995 IEEE Workshop, IEEE Press, New York, 1995.

10 J. Workman Jr., A.W. Springsteen, Applied spectroscopy. A compact reference for practioners, first ed., Academic Press Limited, London, 1998.

11 A. G. Mignani, P.R. Smith, L. Ciaccheri, A. Cimato, S. Graziano, "Spectral Nephelometry for making extravirgin olive oil fingerprints", Sens. Actuator B-Chem. (2003) 157-162.

${ }_{12}$ M. O'Farrell, E. Lewis, W.B. Lyons, N. Jackman, "Combining principal component analysis with an artificial neural network to perform online quality assessment of food as it cooks in a large-scale industrial oven", Sens. Actuator B- Chem. 107 (1) (2005) 104-112. 